\title{
The influence of the magnetic field orientation on the angular momentum loss in the pre-main sequence phase: The case of very slowly rotating magnetic Ap stars
}

\author{
K. Stȩpień ${ }^{1,2}$ and J. D. Landstreet ${ }^{1}$ \\ 1 University of Western Ontario, department of Physics \& Astronomy, London Ontario, Canada N6A 3K7 \\ e-mail: jlandstr@uwo.ca \\ 2 Warsaw University Observatory, Al. Ujazdowskie 4, 00-478 Warszawa, Poland
}

Received 9 November 2001 / Accepted 10 January 2002

\begin{abstract}
Landstreet \& Mathys (2000) have discovered that almost all the magnetic Ap stars having rotation periods longer than about one month have their magnetic and rotation axes fairly closely aligned, in contrast to the more common magnetic Ap stars of shorter period, in which the two axes are usually inclined to one another at a large angle. Furthermore, as shown earlier by Mathys et al. (1997) and by Hubrig et al. (2000), these most slowly rotating magnetic Ap stars have also magnetic fields several times larger than are typical for shorter period magnetic Ap stars, and generally have masses below $3 M_{\odot}$. In this paper, we present calculations to show that the observed axial alignment in the slowest rotators may have arisen during the pre-main sequence (PMS) phase, when stars of a few solar masses are observed as Herbig Ae/Be stars. During the PMS phase, a star is expected to exchange angular momentum with a disk and with a stellar wind. As shown by Stępień (2000), a disk around a PMS magnetic Ap star tends to lock the rotation rate at a few days, while mass loss in the absence of a disk is able to slow the star to quite long periods. We argue that is it plausible to assume that the fraction of the PMS phase during which a disk persists depends on the orientation of the field axis to the plane of the disk, and that disk dissipation may have occurred more quickly in magnetic stars with aligned magnetic and rotation axes than in stars in which the angle between these axes is large. In this case, the magnetic Ap stars with aligned rotation and magnetic axes, especially those with large fields, could lose much more angular momentum than the stars of large obliquity. However, it is also shown that loss of nearly all the angular momentum is only possible for stars with masses below about $3 M_{\odot}$ (and with relatively large fields); for higher masses the available time is too short. Our model thus provides a reasonable explanation for all of the principle observational facts about the very slowly rotating magnetic Ap stars.
\end{abstract}

Key words. stars: chemically peculiar - stars: magnetic fields - stars: pre-main sequence - stars: rotation

\section{Introduction}

It has been known for a long time that the magnetic Ap and Bp stars rotate substantially more slowly than normal stars of the same spectral type (Abt 1979; Wolff 1981; Abt \& Morrell 1995). Stępień (2000, henceforth KS) has recently shown that the slow rotation of magnetic Ap and Bp stars can be accounted for by processes occurring during the pre-main sequence (PMS) phase of stellar evolution. He studied a model of the PMS spin down of a magnetic star which includes accretion of stellar material along the magnetic field lines from an accretion disk, a magnetically coupled interaction between the stellar field and the circumstellar disk, and the effect of stellar mass

Send offprint requests to: K. Stępień,

e-mail: kst@astrouw.edu.pl loss through a magnetized wind. Evolutionary changes of the stellar moment of inertia are included. KS demonstrated that for typically observed values of the accretion rate and mass loss rate due to a wind, significant spin down of a star in the PMS phase occurs provided the star possesses a strong enough magnetic field. The calculated ZAMS rotation periods of the magnetic stars were several times longer than those of normal, non-magnetic stars, assuming the conservation of angular momentum (AM) for the non-magnetic stars.

There exists a group of very slow rotators among Ap stars with the rotation periods of the order of months, years or even tens of years (Mathys et al. 1997). They are too numerous to belong to a slow rotation tail of a single rotation distribution of all magnetic stars (Preston 1970). KS suggested that their rotation periods can be a 
result of a special combination of circumstances during the PMS phase. On the PMS spindown model, very slow rotation is only possible for a star that possesses several characteristics. The star must (a) have a relatively low mass, so that its PMS life time is sufficiently long; (b) it must have a strong magnetic field with a surface intensity of several kilogauss; and (c) it is essential that the accretion disk disappear part way through the PMS phase, while the magnetized wind must continue to operate afterwards for a long enough time.

The theoretical requirements of the PMS spindown model appear to be broadly consistent with observations of the known very slowly rotating magnetic Ap stars. The largest list of magnetic stars of long period is probably that of Mathys et al. (1997), which lists 29 stars for which periods longer than 25 days are established or probable. Of these, 12 are given definite periods there, five more have periods in Landstreet \& Mathys (2000), and 12 still have uncertain (but almost certainly long) periods. One new long-period star (HD 51684) is added to this list by Landstreet \& Mathys. Of these 30 stars, 14 are discussed by Hubrig et al. (2000), where masses are estimated from photometric temperatures and Hipparcos distances.

All of the long-period magnetic Ap stars studied by Hubrig et al. have masses of less than $3.0 M_{\odot}$ (and effective temperatures below $11000 \mathrm{~K}$ ). Of the 16 long-period stars not examined by Hubrig et al., the three hottest (and thus probably highest mass) stars, based on spectral types of B8p, B9p, or A0p, are HD 93507, 114897, and 200311. Their parameters can be derived from the Geneva photometry, together with the Hipparcos parallaxes and the evolutionary tracks given by Schaller et al. (1992). One gets the temperature $9800 \mathrm{~K}$ and mass $2.6-2.9 M_{\odot}$ for HD 93507, $11100 \mathrm{~K}$ and 2.7-3.3 $M_{\odot}$ for HD 144897 (the higher masses are obtained with the Lutz-Kelder correction included), and $13300 \mathrm{~K}$ and $3.4 M_{\odot}$ (without the LK correction) for HD $200311^{1}$. The temperature derived from Geneva photometry for HD 200311 is consistent with $T \approx 12000 \mathrm{~K}$ as estimated by Mégessier (1988) or $13000 \mathrm{~K}$ as estimated by Sokolov (1998). Thus even the hottest known slow rotator, HD 200311, appears to have a mass of less than $4 M_{\odot}$.

Similarly, the magnetic fields of the slowest rotators are clearly almost always unusually large. The mean values of the observed surface field strengths of the slow rotators are tabulated by Mathys et al. (1997). These range from 2.8 to $12.7 \mathrm{kG}$, with a median value of about $5 \mathrm{kG}$. (The hottest of the long-period Ap stars, HD 200311, has a mean surface field of $8.7 \mathrm{kG}$, even larger than the median for the sample.) In contrast, for a sample of nearby normally rotating magnetic Ap stars discussed by Bohlender \& Landstreet (1990), in which the surface fields are not measurable because of rotational line broadening, the median value of the rms longitudinal fields is about $300 \mathrm{G}$, corresponding to a surface field of about $1 \mathrm{kG}$. The fields

\footnotetext{
${ }^{1}$ We thank Dr. Pierre North for providing us these parameters.
}

of the slow rotators thus appear to be typically larger than those of more rapidly rotating Ap stars by a typical factor of about five.

A recent analysis by Landstreet \& Mathys (2000) of the extensive set of measurements of several magnetic field moments for a number of magnetic stars resulted in the unexpected conclusion that almost all the magnetic stars with rotation periods longer than about four weeks have the magnetic and rotation axes roughly aligned (the angle $\beta$ between these two axes is usually smaller than $20^{\circ}$ ), unlike the short period magnetic Ap stars in which the inclination of one axis with respect to the other is usually large $\left(\beta \sim 60^{\circ}\right.$ or more).

The interaction of the stellar magnetic field with the circumstellar matter in the model of KS did not depend on the orientation of the field relative to the stellar rotation axis (which was assumed to be the symmetry axis of the disk). The lifetime of the disk was a free parameter. A relatively short lifetime was assumed for the very slowly rotating Ap stars to let the wind brake the stellar rotation to the observed values. For disks existing during the whole PMS phase, the resulting ZAMS periods could reach, at most, a few weeks.

In the present paper, we extend the model discussed by KS by considering the details of the stellar field-disk interaction. From our considerations, we argue that the orientation of the field influences the disk lifetime. In particular, we argue that disks around the stars with aligned rotation and magnetic axes should exist for about half as long as disks around the stars with strongly inclined axes. As a result, winds efficiently slow down the rotation of aligned (diskless) rotators to the values characteristic of very slowly rotating stars. Inclined rotators can preserve disks for a longer time, hence their ZAMS periods should be, on average, shorter. Thus, the KS model provides a natural explanation for the observed fact that almost all the most slowly rotating magnetic Ap stars have small inclination angles between the rotation and magnetic axes. It is assumed here that the inclination of the field to the rotation axis is approximately constant through the PMS and MS phases, so that presently observed small inclination angles imply small inclinations during the PMS phase. Possible mechanisms for evolutionary changes in inclination angle were discussed by Moss (1990). As his results show, the time scale required for a significant change of the inclination is of the order of the MS lifetime of a star. Furthermore, in the plausible case of a predominantly poloidal internal field, the inclination angle should increase with time. These two results suggest that it is reasonable to assume that presently observed small inclinations imply small inclination angles during the PMS phase.

In Sect. 2 the model of AM loss developed by KS is briefly presented with a modification concerning the conservation of the magnetic field. Section 3 describes the details of the stellar field-disk interaction, following Goodson et al. $(1997,1999)$. It is argued that the rate of removing matter from the disk depends on the orientation of 
the stellar magnetic field. The results of computations of AM evolution of intermediate mass stars under the assumption that the disk exists during the whole PMS phase in strongly inclined rotators and that it exists during only half of the PMS phase in aligned rotators, with a wind operating for the rest of the PMS phase, are presented in Sect. 4. The newest data on variations of the stellar radius and moment of inertia during the PMS evolution, published by Siess et al. (2000) were used. The main conclusions are presented and discussed in Sect. 5 .

\section{Model for $A M$ evolution of a PMS stars with a fossil magnetic field}

The angular velocity of a star possessing a magnetic field changes during the PMS evolution due to the accretion of matter along the magnetic field lines, due to a magnetized stellar wind, due to the interaction of the stellar field with the disk and also because of the change of the stellar moment of inertia. The first three interactions can be described as torques exerted on the star. A more detailed discussion of the torques is given in KS, so here only a brief resumé will be given together with a detailed description of the modifications introduced.

An expression for the disk torque was given by Armitage \& Clarke (1996). We assume, as in KS, that the radius of the magnetosphere (= inner radius of the disk) is equal to the co-rotation radius at which Keplerian angular velocity is equal to stellar angular velocity. The resulting relation for the torque is

$T_{\text {disk }}=-\frac{\mu^{2} \omega^{2}}{3 G M}$,

where $\mu=B_{\text {surf }} R^{3}$ is the magnetic moment of the stellar magnetic field, and $\omega$ is the stellar angular velocity. KS assumed that the fossil magnetic field of an Ap star results in $\mu=$ const. Here we prefer an assumption of a constancy of the total stellar magnetic flux, i.e. $\phi=B_{\text {surf }} R^{2}=$ const (note that, keeping analogy with the definition of $\mu$, a $\pi$ factor is omitted here). We replace $\mu$ with $\phi R$ in the above equation. The disk torque does not depend on mass of the disk as long as the mass exceeds a certain limiting value (Armitage \& Clarke 1996; Cameron et al. 1995). It is assumed here that the disk mass fulfills this condition.

The equation for torque due to accretion was derived by KS assuming that all the AM carried with matter moving from the magnetospheric radius to the stellar surface adds to the stellar AM. In fact this is an overestimate of the AM gain because only a difference between AM of the accreted matter and the stellar surface matter is added to the stellar AM. For slowly rotating stars with relatively strong magnetic field the radius of the magnetosphere is much larger than the stellar radius so this approximation is justified but it is easy to add the term correcting the deposited AM for the specific AM of the stellar matter. The resulting equation for the torque is

$T_{\mathrm{acc}}=\frac{(G M)^{2 / 3} \dot{M}_{\mathrm{acc}}}{\omega^{1 / 3}}-\omega \dot{M}_{\mathrm{acc}} R^{2}$, where $\dot{M}_{\text {acc }}$ denotes the accretion rate. The second term should be multiplied by the sine of the polar angle at which the accreted matter is deposited. We assume that the matter is deposited not far from the equator and we drop that factor.

To calculate the torque due to mass loss, we assume that the wind velocity at the Alfvén surface is equal to the local escape velocity. Adopting a dipolar geometry for the magnetic field, one obtains from the formula (9) in Stępień (1995)

$T_{\text {wind }}=-\frac{\omega R^{7 / 5} \phi^{4 / 5} \dot{M}_{\text {wind }}^{3 / 5}}{3(2 G M)^{1 / 5}}$,

where $\dot{M}_{\text {wind }}$ is the mass loss rate via the magnetized wind.

The final equation for the evolution of the stellar angular velocity is

$$
\begin{aligned}
\frac{\mathrm{d} \omega}{\mathrm{d} t}= & \frac{(G M)^{2 / 3} \dot{M}_{\mathrm{acc}}}{I \omega^{1 / 3}}-\frac{\phi^{2} R^{2} \omega^{2}}{3 I G M} \\
& -\frac{\omega R^{7 / 5} \phi^{4 / 5} \dot{M}_{\mathrm{wind}}^{3 / 5}}{3 I(2 G M)^{1 / 5}}-\frac{\omega \dot{M}_{\mathrm{acc}} R^{2}}{I}-\frac{\omega \mathrm{d} I}{I \mathrm{~d} t}
\end{aligned}
$$

where $I=k^{2} M R^{2}$ is the stellar moment of inertia with $k^{2}$ a radius of gyration.

\section{Magnetic field-disk interaction and the life time of the disk}

The model presented in the previous section assumes a stationary interaction of the stellar magnetic field with the disk. A closer examination of this interaction indicates that this interaction is more likely to consist of separate episodes during which the magnetic field becomes increasingly distorted due to a difference in the rotation rate of the star and the inner part of the disk threaded by the field. The distorted field expands and a reconnection occurs (Lovelace et al. 1995). A plasmoid filled with disk matter moves outwards. At the same time the inner edge of the disk moves towards the star beyond the corotation radius, and the reconnected stellar field drains matter from within the co-rotation radius onto the star. The process repeats at a rate determined by the rate of distortion of the magnetic field, i.e. by the rate of increase of its azimuthal component. When the ratio of this component to the vertical component exceeds a critical value, the reconnection occurs (Lovelace et al. 1995; Goodson et al. 1997).

Goodson et al. (1999) and Goodson \& Winglee (1999) carried out detailed calculations for an aligned dipole, a system with axial symmetry. For a particular choice of the involved parameters, they were able to obtain the frequency of the occurrence of the reconnection events and the amount of matter accreted by the star and expelled into space. The two latter figures turned out to be comparable with one another. Because the expelled matter also carries away disk AM, a self-regulating mechanism of the AM transport exists in the disk: a higher mass loss rate 
means a higher AM loss rate which acts similarly to the increase of the disk diffusivity. In other words, the disk reacts to the higher demand with a higher supply.

Axial symmetry is lost for an inclined dipole. The plasmoids will not have a simple form of torii because the stellar magnetic field will be distorted at a different rate, depending on the azimuthal angle. Close to the plane $S$ containing the dipole axis and perpendicular to the disk plane field lines are perpendicular to the disk plane and the rate of the increase of the toroidal component will be essentially the same as in the case of an aligned dipole. Far from the plane $S$, the field lines are inclined to the disk. In this case only the component of the disk flow perpendicular to the local force line will contribute to its distortion. As a result, the toroidal component of the magnetic field will increase more slowly, and it will need more time to reach the critical value for which reconnection occurs. We can expect now several isolated, local reconnection events, occuring with frequencies determined by the local inclination of the dipole field lines. The dipole magnetic line is inclined to the disk at an angle $90^{\circ}-\beta$. If the interval of time between two consecutive reconnection events is $t_{0}$ for the aligned dipole, we can assume that it is the same close to the plane $S$ but is of the order of $t_{0} / \cos \beta$ at the location $90^{\circ}$ from the plane $S$. The efficiency of removing matter and AM from the disk will decrease from its maximum value on $S$ to this value multiplied by $\cos \beta$ at the location $90^{\circ}$ from $S$. For $\beta=90$ (the magnetic field axis in the plane of the disk) the efficiency will vary smoothly between its maximum value at $S$ and zero. The average value over the azimuthal angle will then be of the order of 0.5 of the maximum efficiency.

We see that the aligned rotator removes matter from the disc about two times more efficiently than the perpendicular rotator. For a given disk mass that means that disks around aligned rotators should have lifetimes about two times shorter than around perpendicular, or strongly inclined rotators. That means, in turn, that winds, on average, have much more time to operate, in the absence of the disk, on aligned rotators. As a result, the aligned rotators should have, on average, substantially longer ZAMS rotation periods, and may reach very long periods. In the next section we will demonstrate that detailed computations confirm this picture. First the input data appropriate for Ap stars and then the results of the detailed computations will be presented.

\section{Input parameters and the results}

\subsection{Input parameters}

It is generally accepted that Herbig AeBe stars are the progenitors of the intermediate mass stars. They are surrounded by disks, and show the presence of intense accretion and stellar outflows. Accretion rates, determined for Herbig AeBe stars of the masses considered here fall in the range $10^{-8}-10^{-6} M_{\odot} \mathrm{yr}^{-1}$ (Hillenbrand et al. 1992), but there exist several apparently disk-less stars, which indicates that disks may be present during only a part of the PMS life (de Winter et al.1997). The estimated mass loss rate of Herbig AeBe stars due to a stellar wind is of the order of $10^{-8} M_{\odot} \mathrm{yr}^{-1}$ (Catala 1989; Palla 1991). Because the intensity of the stellar magnetic field and the lifetime of the disk are primary parameters influencing the ZAMS rotation periods of the magnetic stars, we will not vary accretion rates and mass loss rates due to a stellar wind, although one should remember that the quantitative results depend also on these rates.

Besides the lifetime of the disk, the main parameter needed to solve the problem is the strength of the stellar surface magnetic field. As in KS the standard unit of the magnetic field is taken as value equivalent to a $1 \mathrm{kG}$ field on the surface of a $2 R_{\odot}$ star. The magnetic flux of such a field is equal to $1.9 \times 10^{25}$ in cgs units. Note, however, that, contrary to the assumption made by KS that the magnetic field varies as $R^{-3}$, the assumption of the constant magnetic flux requires that the field varies as $R^{-2}$.

Detailed models of PMS stars of intermediate mass were computed by Iben (1965), by Palla \& Stahler (1993) and very recently by Siess et al. (2000). The latest models show that the PMS phase of these stars lasts somewhat longer that in models obtained by Palla \& Stahler (1993) but not as long as in computations by Iben (1965). Siess et al. (2000) made available on Internet very detailed tables of several stellar parameters as functions of time, including the stellar radius and moment of inertia. These data are used throughout this paper.

The computations of PMS evolution obtained by Siess et al. (2000) start very early, when the star is still within a protostellar cloud. The initial time for the present calculations was adopted as the moment when the star crosses the stellar birth line (Palla \& Stahler 1993). Stars with masses of 3 and $3.5 M_{\odot}$ cross the line twice, so the second time was taken as the initial time. The particular choice of this moment does not influence the main results. Detailed stellar input parameters were taken from Siess et al. (2000) and are given in Table 1.

To allow for changes of the relative importance of the three processes changing stellar AM and described by the first three terms on the right hand side of Eq. (4), efficiency factors were introduced, defined in the same way as in KS

$$
\begin{aligned}
& \dot{M}_{\text {acc }}=k_{\text {acc }} \times 10^{-8} M_{\odot} / \text { year }, \\
& \phi=k_{\text {mag }} \times 1.9 \times 10^{25}, \\
& \dot{M}_{\text {wind }}=k_{\text {wind }} \times 10^{-8} M_{\odot} / \text { year } .
\end{aligned}
$$

These parameters can be regarded as direct measures of the respective parameters entering Eq. (4), e. g. $k_{\mathrm{acc}}=10$ corresponds to the accretion rate $\dot{M}_{\text {acc }}=10^{-7} M_{\odot} \mathrm{yr}^{-1}$, or $k_{\text {mag }}=0.1$ to a surface magnetic field of $100 \mathrm{G}$ on the surface of a $2 R_{\odot}$ star. The surface intensity of the stellar magnetic field on a star with radius $R$ can be calculated from the following formula

$B_{\text {surf }}=\frac{4000 k_{\mathrm{mag}}}{\left(R / R_{\odot}\right)^{2}} \quad \mathrm{G}$. 
Table 1. Stellar input parameters.

\begin{tabular}{ccccccc}
\hline $\begin{array}{c}M \\
\left(M_{\odot}\right)\end{array}$ & $\begin{array}{c}t_{0} \\
\text { (years })\end{array}$ & $\begin{array}{c}t_{\text {ZAMS }} \\
(\text { years })\end{array}$ & $\begin{array}{c}R_{0} \\
\left(R_{\odot}\right)\end{array}$ & $\begin{array}{c}R_{\text {ZAMS }} \\
\left(R_{\odot}\right)\end{array}$ & $\begin{array}{c}I_{0} \\
\left(M_{\odot} R_{\odot}^{2}\right)\end{array}$ & $\begin{array}{c}I_{\text {ZAMS }} \\
\left(M_{\odot} R_{\odot}^{2}\right)\end{array}$ \\
\hline 1.5 & $1.0 \times 10^{6}$ & $4.7 \times 10^{7}$ & 3.10 & 1.44 & 2.85 & 0.13 \\
2.0 & $1.1 \times 10^{6}$ & $2.4 \times 10^{7}$ & 3.48 & 1.62 & 4.72 & 0.23 \\
2.5 & $3.3 \times 10^{6}$ & $1.4 \times 10^{7}$ & 4.30 & 1.81 & 1.84 & 0.39 \\
3.0 & $2.04 \times 10^{6}$ & $8.7 \times 10^{6}$ & 5.54 & 2.00 & 3.95 & 0.59 \\
3.5 & $1.4 \times 10^{6}$ & $6.1 \times 10^{6}$ & 6.71 & 2.19 & 6.91 & 0.87 \\
\hline
\end{tabular}

The three coefficients will be used in the form of a "vector" $\left[k_{\text {acc }}, k_{\text {mag }}\right.$ and $\left.k_{\text {wind }}\right]$ when discussing different solutions.

Equation (4) was solved using a 4th order RungeKutta method, with interpolated values of the stellar radius, the moment of inertia and its derivative calculated from the tables provided by Siess et al. (2000) using cubic spline interpolation (Press et al. 1986). Rigid rotation was adopted, following the assumption that the primordial magnetic field is able to enforce it.

The results of computations will be compared with periods resulting from conservation of AM, henceforth called "normal". These periods do not correspond exactly to periods of non-magnetic stars undergoing the same PMS evolution because disk accretion also spins up a non-magnetic star. The spin up via a boundary layer is, however, usually considerably less efficient than in case of magnetospheric accretion, and is not described correctly by the accretion term in Eq. (4). Comparison of the observed $v \sin i$ values of Herbig AeBe stars with those of their young counterparts on the MS showed that, depending on the degree of AM mixing inside the stars, the two groups have identical or not very different AM (Böhm \& Catala 1995). Errors connected with neglecting any AM change of nonmagnetic stars should therefore be much less than the difference between the ZAMS rotation period of a normal and a magnetic Ap star.

\subsection{Results}

It was demonstrated by KS that each of the mechanisms influencing the stellar AM tends to produce a uniform rotation, independent of the initial value of the rotation period. Our computations confirmed this behavior. Because of this result we will consider only one value of the initial period, equal to 5 days.

While the quantitative results depend on all three efficiency factors $k$, the dependence on the accretion rate and the wind mass loss is of the secondary importance for magnetic stars. The ZAMS period of a star depends primarily on the strength of its magnetic field, assuming the disk persists the whole ZAMS phase or, in case it disappears earlier, the wind disappears simultaneously with it. We fix therefore the value of the accretion rate at $10^{-8} M_{\odot} \mathrm{yr}^{-1}$ (corresponding to $k_{\text {acc }}=1$ ) and the wind mass loss also at $10^{-8} M_{\odot} \mathrm{yr}^{-1}$ (corresponding again to $k_{\text {wind }}=1$ ). We also assume the basic value of $k_{\text {mag }}=5$ as a typical value for a star with strong magnetic field. It corresponds

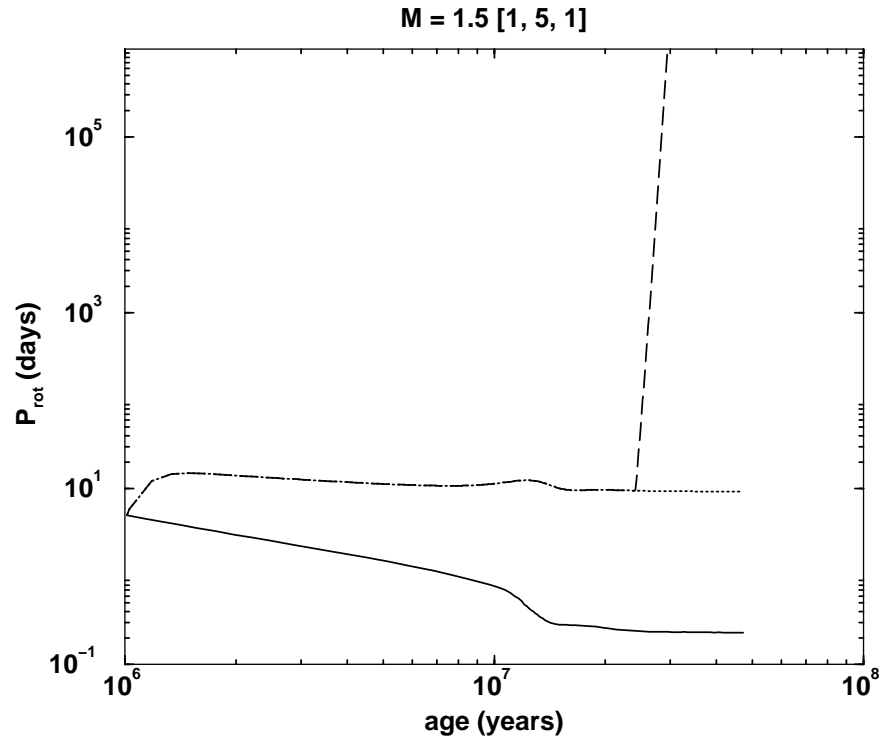

Fig. 1. The importance of the wind in the absence of disk is illustrated for a $1.5 M_{\odot}$ PMS star. For standard values of the parameters, a disk locks the star to a rotation period of about $10 \mathrm{~d}$ (long-dashed line), about 40 times longer than in the non-magnetic case (solid line). If the disk persists to the ZAMS, the period remains at about $10 \mathrm{~d}$ (dotted line). If the disk disappears while the wind remains, the rotation period rises to very large values (short-dashed line).

approximately to $5 \mathrm{kG}$ field (depending on the exact value of the stellar radius) on the surface of a ZAMS star. We will consider the parameter set with $k_{\text {acc }}=1, k_{\text {mag }}=5$ and $k_{\text {wind }}=1$, i.e. $[1,5,1]$, to be our standard set.

Figure 1 demonstrates the importance of the existence of a wind in the absence of the disk. Here, the rotation period evolution is shown for a $1.5 M_{\odot}$ star with the standard set of efficiency factors. A dotted line corresponds to the case when the disk exists the entire PMS phase. The ZAMS rotation period is equal to about 10 days, i.e. 40 times longer than in the non-magnetic case, when the AM is conserved (a solid line - the ZAMS period is 0.23 day). A dashed line corresponds to a situation when the disk disappears after the first half of the stellar PMS life but the wind operates till ZAMS. The resulting rotation period is very long - in fact its value is a high power of ten. Even with the magnetic field decreased by a factor of two and the wind mass loss by a factor of ten the ZAMS period in the case of the wind operating on the disk-less star of this mass produces a very long ZAMS 


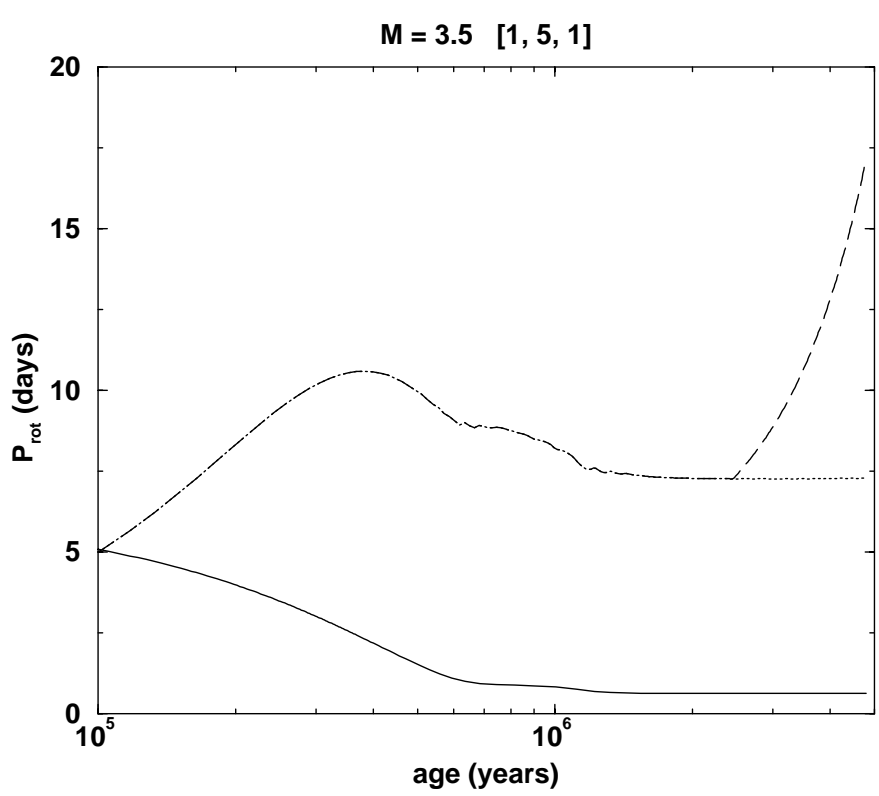

Fig. 2. The wind becomes unimportant for massive stars. The evolution of the rotation period is shown for a $3.5 M_{\odot}$ star. As in the previous figure, the disk locks the star into a rotation period of several days (dot-dash line) which becomes the ZAMS period if the disk is present throughout the PMS phase (dotted line). If the disk vanishes half-way through the PMS lifetime, the period only has time to increase by a little more than a factor of two (long-dash line). Again, the non-magnetic star reaches a much shorter rotation period than the magnetic star (solid line).

period of the order of 70 years. In the presence of the strong magnetic field, the disk locks the stellar rotation period to a sort of equilibrium value which remains constant as long as the disk exists. If the disk disappears, but the wind persists, the wind efficiently brakes the star. The resulting rotation period depends on the ratio of the wind timescale for slowing down to the stellar PMS timescale. The wind timescale is of the order of $3 \times 10^{5}$ years for the $1.5 M_{\odot}$ (see Eq. (4)), but it increases quite rapidly, to about $2 \times 10^{6}$ years for a $3.5 M_{\odot}$, mainly due to the increase of the moment of inertia with increasing mass (Table 1). The PMS lifetime decreases with increasing mass: it is about $4.6 \times 10^{7}$ years for the $1.5 M_{\odot}$, i.e. two orders of magnitude longer than the the wind timescale, but it is $6 \times 10^{6}$ years for the $3.5 M_{\odot}$, which is merely 3 times more than the wind timescale for this star. Figure 2 shows that the wind is, indeed, inefficient in the case of this more massive star. If the disk disappears after the first half of the PMS lifetime but the wind operates for the rest of the PMS phase, the ZAMS period is equal to 17 days i.e. only 2.5 times more than in the case in which the disk exists during the whole PMS phase. A star of such a high mass cannot reach a very long rotation period of the order of years.

The results of similar computations for all the stellar masses considered are given in Fig. 3. A solid line shows the dependence of the ZAMS rotation period on stellar mass for the situation when the disk is switched off

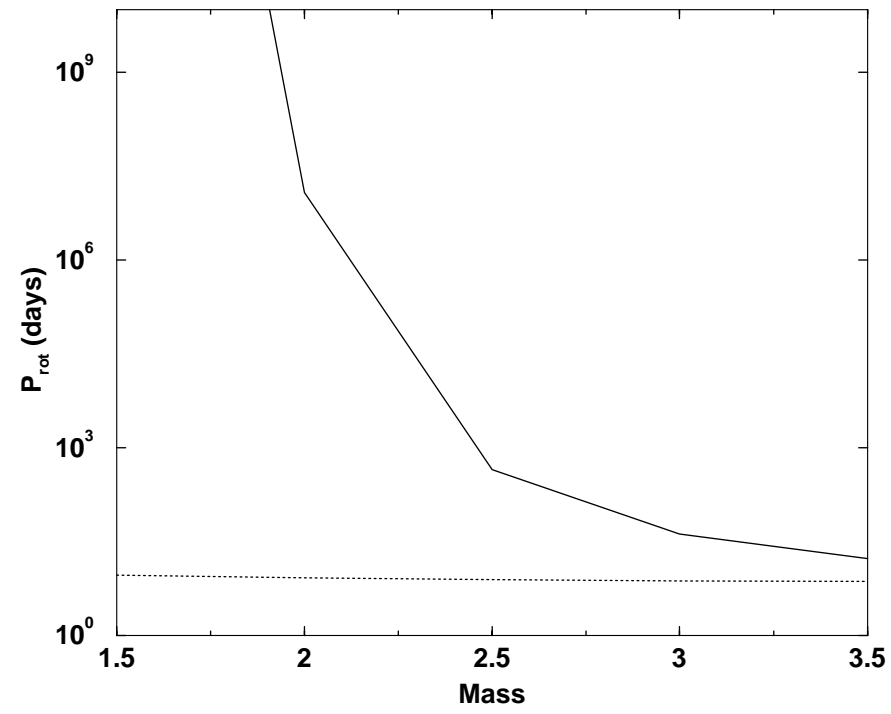

Fig. 3. The ZAMS period achieved as a function of stellar mass for models with the standard parameter set, for the case in which the disk disappears after half the PMS lifetime (solid line) and for the case in which the disk remains throughout the PMS evolution (dotted line).

after the first half of the stellar PMS life and the wind persists till ZAMS. The standard set of efficiency factors was used. A dotted line shows the ZAMS period when the disk persists for the whole PMS phase. We see a very rapid decrease of the period resulting from the operation of the wind alone with increasing mass. For masses exceeding $2.5-3 M_{\odot}$ the longest expected period is at most of the order of a few weeks. We draw the conclusion that only less massive stars can attain very long rotation periods, whereas we should not see slowly rotating stars with masses above about $3 M_{\odot}$. A precise value for the limiting mass of slow rotators may vary with detailed values for some of the parameters considered, but it should not be much different from our estimate because it is a result of the crossing of two functions strongly dependent on mass: one is the PMS lifetime, steeply decreasing with increasing mass and the other is the wind slow down timescale, steeply increasing with increasing mass. The first function is completely independent of our model, whereas the second depends relatively weakly on the parameters of the present model and its strong dependence on mass is the result of the fact that the stellar moment of inertia varies strongly with mass (Table 1 ).

The observed magnetic field varies substantially from one Ap star to another. It is interesting to see how the ZAMS period depends on its particular value. As an example, Fig. 4 shows the dependence of the ZAMS rotation period on the intensity of the stellar magnetic field for a $2.5 M_{\odot}$ star. Dashed lines describe the evolution of the rotation period when the disk exists the whole PMS phase and dotted lines correspond to the situation when the wind operates on a disk-less star for the last half of the stellar PMS life. Values of $k_{\text {mag }}$, shown in the figure, describe the curves in the same order, from the top to 


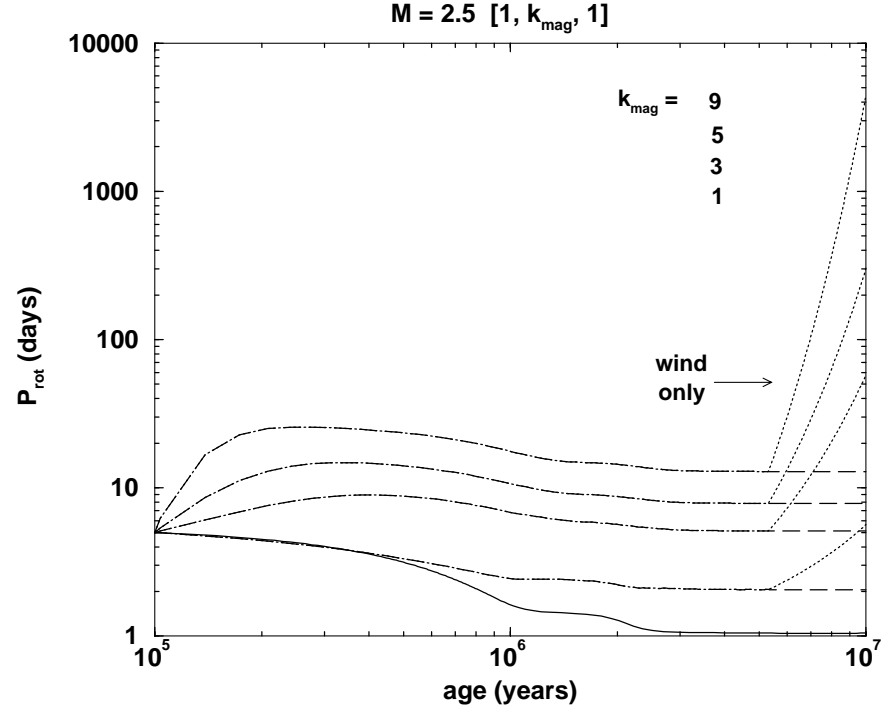

Fig. 4. The ZAMS rotation period depends on the intensity of the stellar magnetic field. For a $2.5 M_{\odot}$ model, the period evolution is shown for several values of the magnetic parameter $k_{\text {mag }}$ (from top to bottom, 9, 5, 3, and 1) in the case of the disk persisting (dot-dash then long-dash lines) and in the case of the disk vanishing after half the PMS lifetime (dot-dash then dotted lines). Again the non-magnetic model (solid line) evolves to a much shorter period.

bottom. For comparison a non-magnetic case is shown as a solid line. The ZAMS period corresponding to this curve is equal to about 1 day. As expected, the ZAMS rotation period increases with an increase of the magnetic field but, again, with the disk existing during the whole PMS phase the longest expected periods are of the order of one or two weeks, even for strong magnetic fields. A very slow rotation can be attained only with the wind in the absence of disk. A somewhat puzzling aspect of the observations in this connection has been noted by Mathys et al. (1997), who point out that about half the long-period magnetic Ap stars with periods shorter than 150 d have average values of the mean surface field in excess of $7.5 \mathrm{kG}$, while none of the slowest rotators do.

\section{Discussion and conclusions}

We may summarize our results as follows. We are trying to understand the origin of the small population of magnetic Ap stars which have very long rotation periods (longer than about $25 \mathrm{~d}$ ), and thus unusually small specific angular momentum, both with respect to normal $\mathrm{A}$ and $B$ stars, and with respect to the large majority of magnetic Ap stars with periods in the range of $0.5-25 \mathrm{~d}$. The properties of these very slowly rotating magnetic Ap stars that are relevant are the following:

- These stars have magnetic fields which are typically several times larger than are found in more rapidly rotating magnetic Ap stars.
- The very slowly rotating magnetic Ap stars have magnetic field structures in which the dipolar axis is typically aligned to within about $20^{\circ}$ of the rotation axis, in complete contrast to the situation for the great majority of magnetic Ap stars, in which the two axes are usually $60^{\circ}$ or more apart.

- The known very slow rotators virtually all have masses below $3 M_{\odot}$. None of the numerous more massive magnetic B stars have periods of more than a few weeks (see e.g. Bohlender et al. 1993).

As discussed in more detail in KS, the evidence is fairly clear that magnetic Ap stars achieve their unusually low specific angular momentum (with respect to normal A and B stars) prior to the main sequence stage. Probably the strongest evidence for this is the fact that the angular momentum distribution of young magnetic Ap stars is similar to that of older ones. Furthermore, a wind strong enough to carry off most of the stellar angular momentum during the main sequence phase would overwhelm diffusion, leading to spectral normality; but there is no evidence of global magnetic fields in any normal A or B stars (Landstreet 1982; Shorlin et al. 2002) with the exception of one magnetic Be star, $\beta$ Cep (Donati et al. 2001). We thus place the epoch of angular momentum loss prior to the arrival on the ZAMS. The fact that magnetic Ap stars form in clusters along with stars of normal specific angular momentum indicates that the origin of slow and very slow rotation is not in the angular momentum of the parent cloud, but something specific to individual stars. This leads us to consider mechanisms that may occur during the PMS phase (the Herbig Ae/Be stage), which is observationally characterized by the presence of disks and winds.

Previous computations carried out by KS have shown that if a disk is present around a PMS magnetic star right up until it reaches the ZAMS (and the wind dies away), the rotation of the star is locked into a period of a few days, as is observed for the great majority of magnetic Ap stars. In order to lose most of its remaining angular momentum, a star must lose its disk but continue to have a magnetically coupled wind for about another $10^{7} \mathrm{yr}$ or more. This suggests that the difference between the common slowly rotating magnetic Ap stars and the very slowly rotating ones may be a difference in the time required to dissipate the disk. The fact that these two categories of stars have quite different magnetic configurations suggests that the time required for disk dissipation may be a function of magnetic field structure.

If we make the assumption (justified in Sect. 2 above) that disk dissipation occurs in about half the PMS contraction time in stars in which the field and rotation axis are aligned, but requires nearly the full PMS phase in stars with large field inclinations, our model calculation indicates that indeed very long periods may be reached by aligned magnetic rotators. However, these long periods can only be achieved by stars whose masses do not significantly exceed $3 M_{\odot}$; in more massive stars the wind torque 
is not high enough to slow the stars to such long periods in the available PMS timescale. Furthermore, the slowing is expected to depend reasonably strongly on the global magnetic field strength; it is not at all surprising that the observed very slow rotators have fields that are typically several times larger than the median field found in more rapidly rotating magnetic Ap stars. Thus, simple, plausible assumptions about how angular momentum loss occurs in PMS stars, and how this process depends on field structure, are sufficient to account for all the salient features of the very slowly rotating sample of magnetic Ap stars.

Direct tests of this theory through observations of PMS stars are not yet possible. Although a large number of PMS A and B stars (Herbig Ae/Be stars) are known, little is yet known observationally about the occurrences of magnetism in such stars. A search for magnetic fields among Herbig Ae/Be stars, carried out by Glagolevskij \& Choutonov (1998), brought null results within the accuracy of measurements but Donati et al. (1997) report a detection of a field in HD 104237; see also http://webast.ast.obs-mip.fr/people/donati/ field.html). A considerably larger sample of field detections in PMS stars will be needed before we can observationally determine whether disk longevity is related to magnetic obliquity, as we postulate.

Acknowledgements. We thank Dr. P. North, the referee, for helpful remarks. This work has been supported in part by the Natural Sciences and Engineering Research Council of Canada. Partial support from KBN grant 5P03D 00621 is also acknowledged.

\section{References}

Abt, H. A. 1979, ApJ, 230, 485

Abt, H. A., \& Morrell, N. J. 1995, ApJS, 99, 135

Armitage, P. J., \& Clarke, C. J. 1996, MNRAS, 280, 458

Bohlender, D. A., \& Landstreet, J. D. 1990, MNRAS, 247, 606

Bohlender, D. A., Landstreet, J. D., \& Thompson, I. B. 1993, A\&A, 269, 355

Böhm, T., \& Catala, C. 1995, A\&A, 301, 155

Cameron, A. C., Campbell, C. G., \& Quaintrell, H. 1995, A\&A, 298,133
Catala, C. 1989, in Low mass star formation and pre-MainSequence evolution, ed. Bo. Reipurth (ESO, München), 471

Donati, J.-F., Semel, M., Carter, B. D., Rees, D. E., \& Cameron, A. C. 1997, MNRAS, 291, 658

Donati, J.-F., Wade, G. A., Babel, J., et al. 2001, MNRAS, 326, 1265

Glagolevskij, Yu. V., \& Choutonov, G. A. 1998, Bull. Spec. Astrophys. Obs., 45, 105

Goodson, A. P., Böm, K.-H., \& Winglee, R. M. 1997, ApJ, 489, 390

Goodson, A. P., Böm, K.-H., \& Winglee, R. M. 1999, ApJ, 524, 142

Goodson, A. P., \& Winglee, R. M. 1999, ApJ, 524, 159

Hillenbrand, L. A., Strom, S. E., Vrba, F. J., \& Keene, J. 1992, ApJ, 397, 613

Hubrig, S., North, P., \& Mathys, G. 2000, ApJ, 539, 352

Iben, I. 1965, ApJ, 141, 993

Landstreet, J. D. 1982, ApJ, 258, 639

Landstreet, J. D., \& Mathys, G. 2000, A\&A, 359, 213

Lovelace, R. V. E., Romanova, M. M., \& Bisnovatyi-Kogan, G. S. 1995, MNRAS, 275, 244

Mathys, G., Hubrig, S., Landstreet, J. D., Lanz, T., \& Manfroid, J. 1997, A\&AS, 123, 353

Mégessier, C. 1988, A\&AS, 72, 551

Mestel, L., \& Takhar, H. S. 1972, MNRAS, 156, 419

Moss, D. L. 1990, MNRAS, 244, 272

Palla, F. 1991, in Fragmentation of molecular clouds and star formation, ed. K. Falgerone et al. (Kluwer, Dordrecht), 331

Palla, F., \& Stahler, S. W. 1993, ApJ, 418, 414

Press, W. H., Teukolsky, S. A., Vetterling, W. T., \& Flannery B. P. 1986, Numerical Recipes (Cambridge University Press, Cambridge)

Preston, G. W. 1970, in Stellar Rotation, ed. A. Sletteback (Reidel, Dordrecht), 254

Schaller, G., Schaerer, D., Meynet, G., \& Maeder, A. 1992, A\&AS, 96, 269

Siess, L., Dufour, E., \& Forestini, M. 2000, A\&A, 358, 593

Shorlin, S., Wade, G. A., Donati, J.-F., et al. 2002, in preparation

Sokolov, N. A. 1998, A\&AS, 130, 215

Stępień, K. 1995, MNRAS, 274, 1019

Stȩpień, K. 2000, A\&A, 353, 227 (KS)

de Winter, D., Koulis, C., Thé, P. S., et al. 1997, A\&AS, 121, 223

Wolff, S. C. 1981, ApJ, 224, 221 